\title{
Leakage Flux Modeling of Multi-Winding Transformers for System-Level Simulations
}

\author{
Min Luo, Member, IEEE, Drazen Dujic, Senior Member, IEEE, and Jost Allmeling, Member, IEEE
}

\begin{abstract}
In multi-winding transformers, different geometry of the individual windings leads to unbalanced leakage flux paths. The unbalance affects the behaviour of the power electronic converters, where these transformers are used. This work proposes an approach to model the leakage flux path of transformer with repetitive multi-winding structure using permeance magnetic circuit. The model is composed of lumped components, it can be seamlessly integrated into system-level simulation of power electronic circuits and achieve good accuracy in time-domain simulation. Taking advantage of the repetitive structure, the model requires very limited number of parameters, which can be easily obtained from the geometry information together with only a few experimental tests. The fidelity of the model is experimentally confirmed on a multi-winding transformer prototype connected to power electronic devices.
\end{abstract}

Index Terms-leakage flux modeling, dynamic, system-level simulation, magnetic circuit, permeance-capacitance

\section{NOMENCLATURE}

$W_{p} \quad$ Primary winding.

$N_{p} \quad$ Primary winding number of turns.

$R_{p} \quad$ Primary winding resistance.

$U_{p} \quad$ Primary winding voltage.

$W_{s} \quad$ Secondary windings.

$N_{s} \quad$ Secondary winding number of turns.

$R_{s} \quad$ Secondary winding resistance.

$U_{s} \quad$ Voltage on the secondary windings.

$I_{s} \quad$ Current on the secondary windings.

\section{INTRODUCTION}

$\mathbf{M}$ ULTI-WINDING transformers have been applied in power electronic converters to interface systems operating on different voltage levels. In typical multilayer- (Fig. 1(a)), multidisk- (Fig. 1(b)) and mixed- (Fig. 1(c)) structures, non-identical size and position of the individual windings may lead to different leakage flux coupling, which gives rise to unbalanced short-circuit impedances [1]. This unbalance is directly reflected in the behaviour of the converter system. To investigate any potentially negative affect of the unbalance, and subsequently adapt component selection for the rest part of system or explore solutions by means of control algorithm, sufficiently accurate transformer model which can be combined with power electronic circuit for system-level simulation is desired.

M. Luo and D. Dujic are with the Power Electronics Laboratory, École Polytechnique Fédérale de Lausanne (EPFL), Lausanne, CH-1015 Switzerland (e-mail:min.luo@epfl.ch,drazen.dujic@epfl.ch).

J. Allmeling is with Plexim GmbH, Zürich, CH-8005 Switzerland (e-mail: allmeling@plexim.com).

Manuscript received December 19, 2016; revised Feb 13, 2017; accepted April 11, 2017.

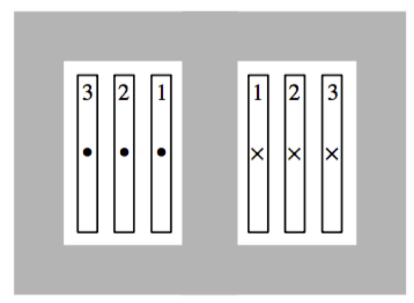

(a)

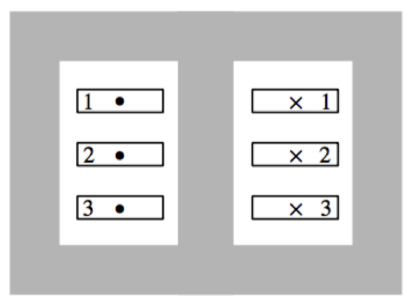

(b)

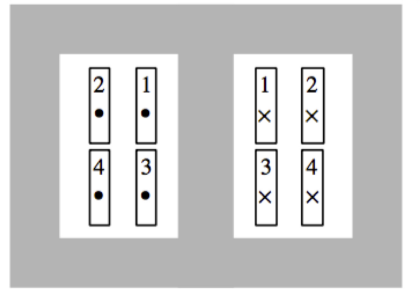

(c)

Fig. 1: Winding arrangements considered in the existing publications [9] [10] [11][13], (a) Multilayer (b) Multidisk (c) Mixed

Finite element method (FEM) has been widely adopted in modelling the main- and leakage flux distribution. However FEM is not suitable for simulation combined with complex power electronic circuits, due to its high computational effort and poor convergence in these circumstances. For systemlevel simulation, transformers are commonly represented by electrical equivalent circuit using coupled inductors. Coupledinductor approach requires electric test to identify all the selfand mutual inductance values including the main flux path $L_{m}$ and leakage flux path $L_{\sigma}$. If more than two windings are present, electric test needs to be enumerated among different short-and open circuit combinations, which can be time consuming and in some cases impractical.

Besides FEM and coupled-inductor, magnetic circuit has become popular nowadays. Firstly, the complexity is much lower than FEM thanks to the lumped representation, also the integration to electrical circuit is nearly seamless. Secondly, magnetic circuit has closer relation to the geometry than coupled-inductor, making it possible to parametrise the flux path directly without any electric tests. In combination with the material characteristic, this methodology has been successfully applied to model the main flux path [2] [3] [4] [5] [6]. However the leakage flux path was not analysed.

Difficulty exists in the parametrisation of leakage flux path in magnetic circuit. One of the underlying assumption in 
magnetic circuit approach is that the fluxes are confined in virtual tubes. Each section of the tube is characterised by the geometry together with material's permeability $\mu$ as lumped reluctance or permeance. Parametrising the main flux path through the iron core is straightforward, since the geometry is determined and directly visible. In comparison however, the geometry of the leakage flux path through the air is usually not clearly shaped.

In the work of [7] permeances representing leakage flux path has been introduced into the magnetic circuit of transformer with closed pot-type core. Thanks to the special core-structure, geometry of the leakage flux path was well determined, so that parametrising could be done in similar manner as the main flux path. However this type of transformer is not typically adopted in high-power applications. For typical Ecore structure, authors of [8] have made a clear classification for leakage flux paths, and parametrised the permeances by geometrical data. However, this approach has been verified only on a single-winding inductor, where no leakage coupling between different windings takes place as in transformers.

For transformers with typical core structure, several publications have successfully modelled the leakage flux path. In the work of [9], a generalised reluctance magnetic circuit including leakage flux path between the windings was presented for complex multi-winding structures. Authors of [10] and [11] have followed this approach for simulation of different types of transformer. However in all these publications the magnetic circuit served as a intermediate step to derive the terminal-duality model (TDM) introduced by [12], which was essentially electrical equivalent circuit. In TDM the main flux path was represented by shunt-inductors on winding terminals, while the leakage flux path by coupled leakage inductance network in between. Elements of the leakage inductance matrix are linear summation of the short-circuit impedances between certain winding pairs, requiring again large number of electric tests. Especially when the leakage inductance matrix is being composed by measured short circuit impedance, assumption has been made that the inductance of the main flux path is magnitudes higher, which is not always the case in reality. Moreover, since the information about geometry is hardly visible after conversion to TDM model, open-circuit test is still necessary to identify the main inductance on winding terminals and this may make the representation of the material nonlinearity less accurate. As extension to the work done by [9], authors of [13] calculated the short-circuit impedances between winding pairs directly from geometrical data using the method introduced in [14], to get rid of the electric tests. The simulated short-circuit impedance matched well to the experimental measurements from single- and three phase transformers. However the increased error in three-phase case indicates that the 2D formulation of the method from [14] may run into issues, if the part of windings outside the core window area dominates.

The leakage flux models with direct parametrization from geometry are difficult to generalize in terms of accuracy, while the ones parametrised from electric test do not include the geometrical information contained in the magnetic circuit, so that large measurement effort is needed. This work proposes

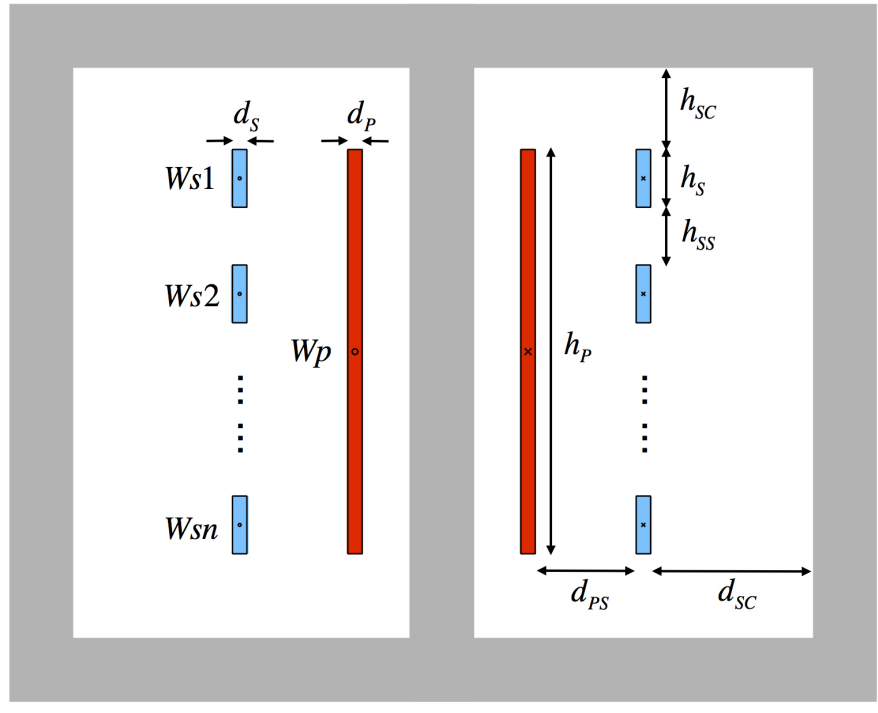

Fig. 2: Winding arrangement with long primary winding and multiple stacked secondary windings

a solution which combines the intuitive geometric information and experimental results from only a few electric tests together, and is able to achieve good accuracy, as demonstrated in the paper.

In most of the complex transformers, the individual windings do have something geometrically in common, due to consideration of mass production or space utilisation. Taking advantage of this repetitive feature, this paper will demonstrate how the leakage flux path model and its parametrisation procedure can be simplified, meanwhile demonstrating that the proposed approach of model is able to reproduce the unbalance of short-circuit impedance in reality. It is an extension of the work done in [15] and distinguished from the other previous publications via combining the following aspects together:

- Magnetic circuit is directly simulated in its original form, without transformation into electrical equivalent. The main- and leakage flux paths related to the real geometrical structure are contained in the same model and observable during the simulation.

- Main flux path is parametrised directly from geometry. Model of material nonlinearity like hysteresis proposed by [16] and corner flux concentration, analysed in [17], can be potentially incorporated, but are not analysed in this paper.

- Leakage flux path is characterised by a few parameters only, identification of which can be done via fitting to only a small number of electric test. The influence of the main flux path has been taken into account, apart from the infinite permeability assumption made in [9].

Typical winding arrangements in Fig. 1(a), Fig. 1(b) and Fig. 1(c) have been investigated in the existing publications [9], [10], [11] and [13], while the one depicted in Fig. 2 has not been discussed in detail so far, where the height of the inner layer winding spread out the total height of the others. This arrangement is typical for the phase-shift transformers used in multi-pulse rectifier systems, which interface power 
grid to medium voltage power electronic converters like the cascaded H-bridge patented by Robicon Corporation [18] and widely applied in industry nowadays. Simplified single-phase transformer version of this winding arrangement is taken for the case study presented in the paper. A low power prototype transformer has been constructed to evaluate fidelity of the proposed modelling's approach. Besides the verification scheme of supplying one winding and short-circuit another, which has been carried out in the existing publications including [15], more short-circuit combinations as well as connection to power electronic converters are evaluated in this work, to verify if the model has sufficiently covered all kinds of coupling through leakage flux path. The permeance-capacitor element proposed by [19] and implemented by [20] is chosen to compose the magnetic circuit, considering its capability to apply ordinary differential equation (ODE) solver which is already provided in numerous commercial system-level simulation softwares like Simulink and PLECS.

This paper is organised as follows. Section II demonstrates the modelling's approach as well as the procedure for parameter identification. Section III provides the verification of the fidelity of the model via different experimental short-circuit tests. Afterwards in Section IV, the fidelity of the model in system-level simulation is further evaluated in combination with power electronic converters. Finally, Section V concludes the work and provides outlook for future improvements.

\section{MODELING}

The prototype transformer depicted in Fig. 3 has been taken as study case. This transformer is rated to convert $115 \mathrm{Vrms}$ $400 \mathrm{~Hz}$ low voltage to $15.2 \mathrm{Vrms}$ on nine output terminals with nominal power of $180 \mathrm{~W}$ in total. Each output terminal can be connected to full-bridge rectifier to generate $20 \mathrm{~V} \mathrm{DC}$ voltage as input for switched-mode power supplies. The iron core is stacked by laminations type "EI 150/150" of material "M330-35A", resulting in total thickness of $D_{C}=50 \mathrm{~mm}$. There is one primary winding $\left(W_{p}\right)$ with $N_{p}=144$ turns on the input side and nine secondary windings $\left(W_{s 1} \ldots W_{s 9}\right)$ with $N_{s}=19$ turns each on the output side. Low window area utilisation is configured for this prototype transformer in order to exaggerate the leakage flux path and resemble the high winding spacing in medium voltage applications due to consideration of insulation.

TABLE I: Dimensional parameters of the prototype transformer in [mm]

\begin{tabular}{c|c|c|c}
\hline Parameter & Value & Parameter & Value \\
\hline$d_{P}$ & 2 & $d_{S C}$ & 18 \\
\hline$h_{P}$ & 71 & $h_{S C}$ & 9 \\
\hline$d_{S}$ & 3 & $d_{P S}$ & 3 \\
\hline$h_{S}$ & 7 & $h_{S S}$ & 1 \\
\hline
\end{tabular}

The windings of the prototype transformer have been arranged complying to the structure in Fig. 2. All dimensional parameters have been listed in TABLE I. Typical geometrical repetition and symmetry present in complex transformers has been introduced to the prototype. All secondary windings $W_{s k}$

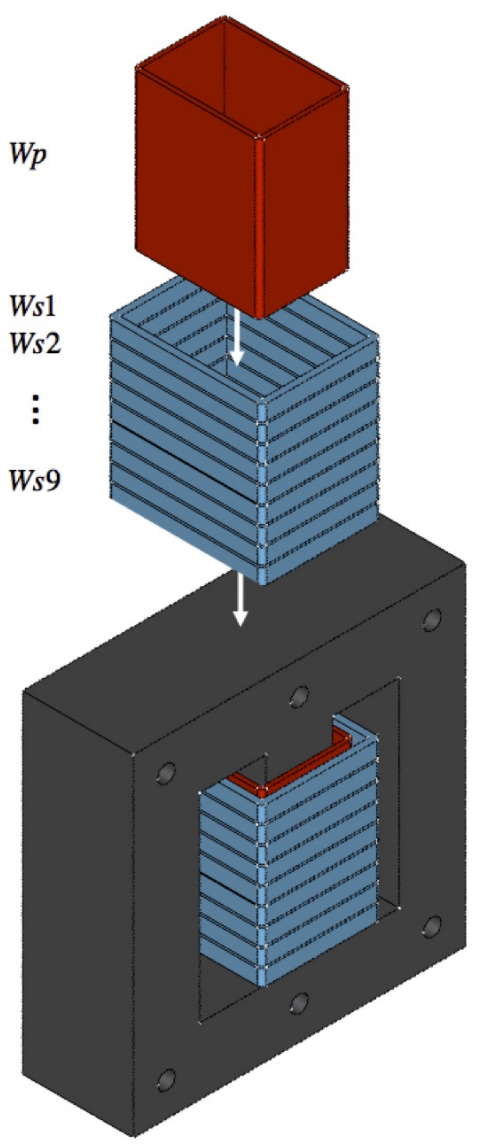

Fig. 3: Structure of the prototype transformer with one primary- $W_{p}$ and nine secondary windings $W_{s k}$

$(k=1 \ldots 9)$ are designed to be the same size, which are allocated along the middle core limb with the same vertical interval in between. Please note that $h_{S S}$ is significantly smaller than the winding's dimension. The primary winding $W_{p}$ is placed inside the secondary winding, whose height is equal to the distance from the top of $W_{s 1}$ to the bottom of $W_{s 9}$.

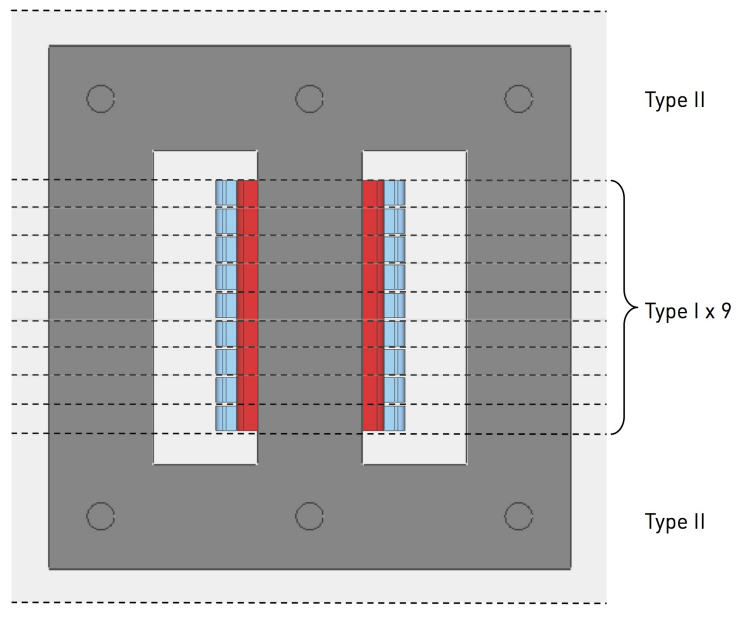

Fig. 4: Vertical separation of the transformer's geometry into nine divisions of type I and two of type II 


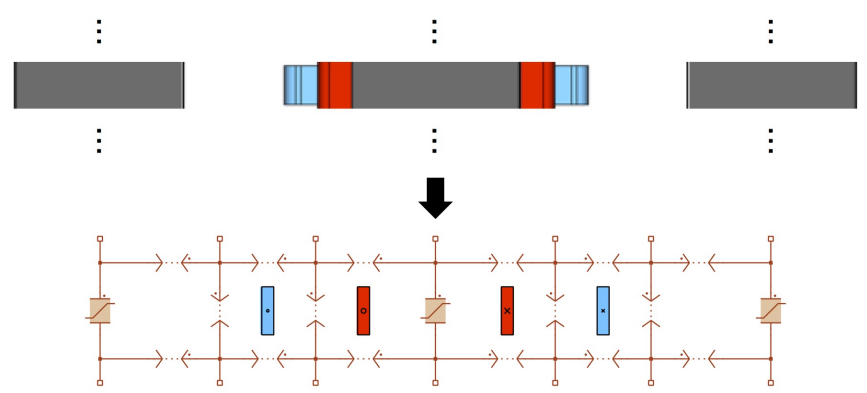

Fig. 5: Permeance magnetic circuit of the division type I with the flux path of the core and air

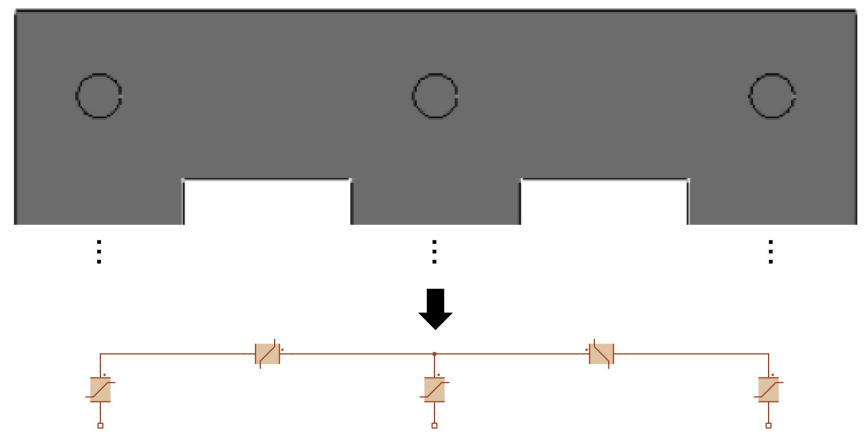

Fig. 6: Permeance magnetic circuit of the division type II

To derive the magnetic circuit model, the geometry of the transformer is separated into divisions along the vertical axis, as Fig. 4 demonstrates. Nine divisions of type I have been defined. All of them have the same height equal to $h_{\text {DivI }}=h_{S}+h_{S S}$, each one includes one section of the three core limbs, one complete secondary winding, as well as one section of the primary winding. The turns number of the primary winding section is equal to $N_{p D i v}=N_{p} / 9$. This division can be converted into a magnetic circuit shown in Fig. 5 . The three permeances filled with solid color represent flux path inside the transformer core, while the others represent the leakage flux path through the air. The cross section of the windings are placed schematically in between, according to the real geometry.

Besides that, two divisions of type II representing the rest of the transformer is demonstrated in Fig. 6, including core yoke as well as the top and bottom part of the limbs. In comparison to the division type I, since no winding is present in division type II and the permeability of the main flux path inside the core is much more dominant than that of the leakage flux path through the air, and the leakage permeances in this type of division can be neglected.

Stacking nine divisions of type I over each other and adding two divisions of type II on the top and bottom, respectively, yields the complete magnetic circuit as shown in Fig. 7. If all the windings are installed close enough to the middle limb of the transformer core (as is usually the case in practice to reduce leakage inductance), the horizontal leakage permeances aboveor below the windings can be assumed to be "magnetically" short circuit, resulting in the structure of Fig. 8. The other horizontal permeances stand for the leakage flux paths from

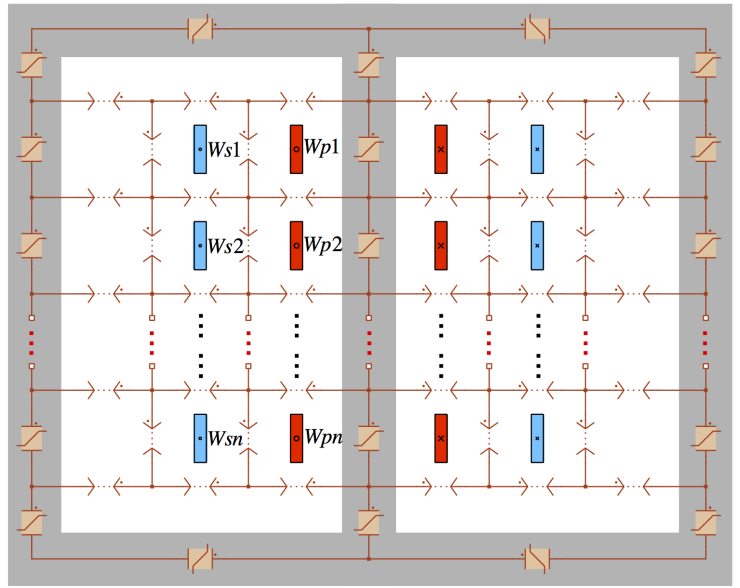

Fig. 7: Original permeance circuit of the transformer with schematically placed windings

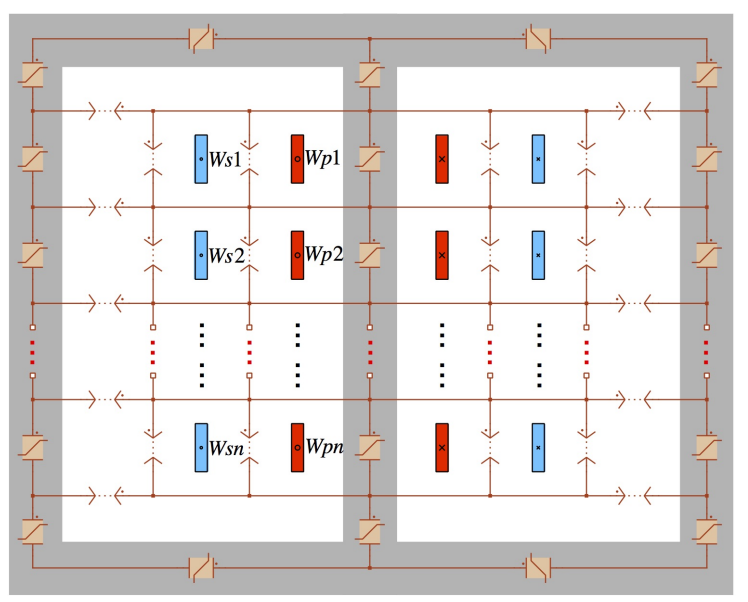

Fig. 8: Permeance circuit of the transformer with the horizontal leakage permeances above- or below the windings neglected

middle- towards side limb, while the vertical ones surrounding $W_{p}$ and $W_{s}$ for the leakage flux paths looping back to the middle limb. Also one should keep in mind that these permeances account not only for the leakage flux paths inside the core window area, but also the ones exposed outside the middle limb (e.g. fringing field), despite of the 2-D sketches being used for illustration.

Afterwards, the schematically depicted windings are converted into lumped-components as demonstrated in Fig. 9, which is ready to be directly connected to electrical circuit model. The lumped winding components are implemented using the gyrator structure proposed by [19], which serves as interface between the electrical and magnetic circuit shown in Fig. 11. In one direction, the electrical voltage measured on the winding terminal is divided by the number of turns $N$ and fed into the magnetic circuit as a flux rate source $(d \Phi / d t)$. The flux rate source "charges" or "discharges" the magnetic permeances which behave like capacitors (not electrical). In the other direction, the "magnetic voltage" measured on the magnetic terminal (magnetomotive force " $M M F$ ") is also divided by $N$ and fed into the electrical circuit as electrical current source. 


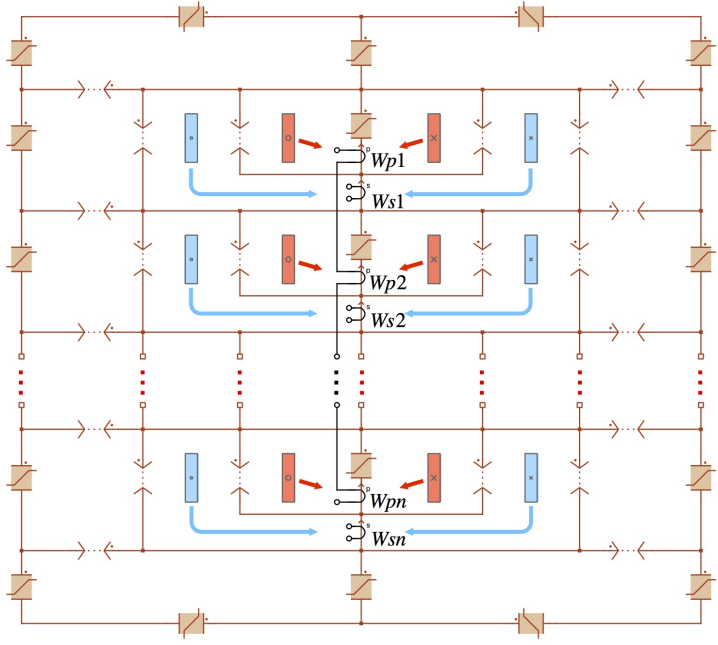

Fig. 9: Permeance circuit with lumped-winding components to interface electrical circuit

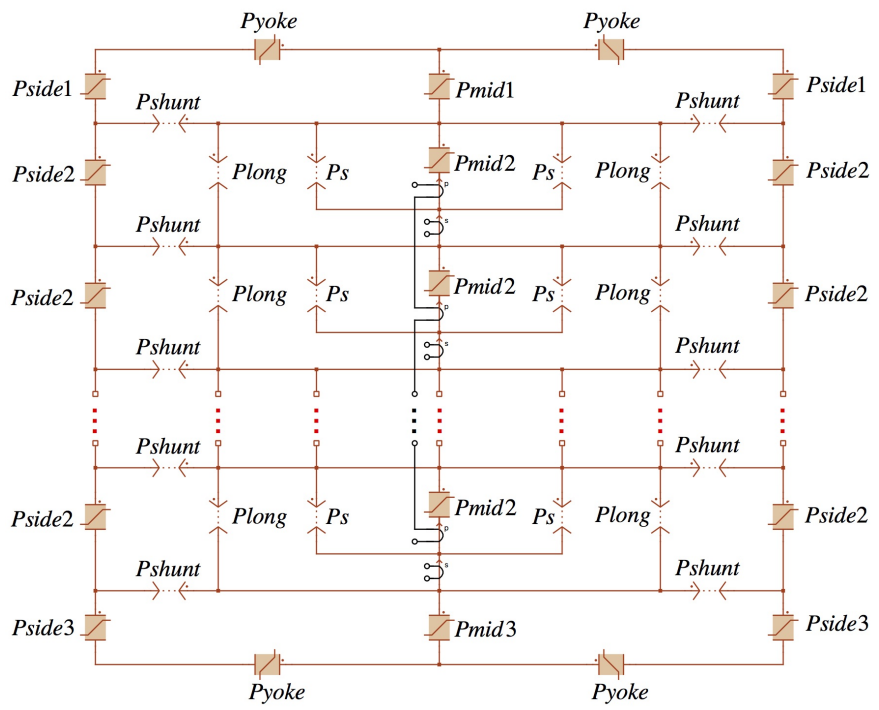

Fig. 10: Final version of the magnetic circuit model

Please note that all the primary winding sections are seriesconnected in the electrical circuit, so that all sections share the same electrical current while the terminal voltage is the summation of all the individual voltages. For this prototype transformer, the final version becomes the structure in Fig. 10.

The permeances representing different part of the iron core can be calculated as:

$$
P=\frac{\mu_{r} \mu_{0} A}{l}
$$

In the equation above, the equivalent cross-section area $A$ and magnetic path length $l$ can be calculated from the core geometry using the formulas introduced in [21]. For the prototype transformer, the length of $P_{m i d 2}$ and $P_{s i d e 2}$ is equal to $h_{S}+h_{S S}$, while that of $P_{m i d 1}\left(P_{m i d 3}\right)$ and $P_{\text {side } 1}\left(P_{\text {side } 3}\right)$ equal to $h_{S C}$ (geometry parameters see Fig. 2). The permeance $P_{\text {yoke }}$ includes both the yoke and corner. Should nonlinearity

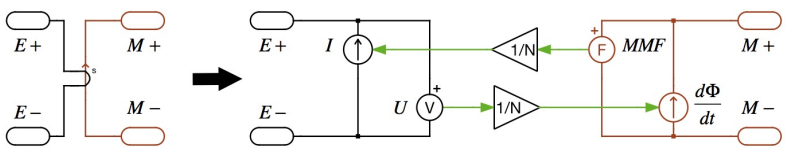

Fig. 11: Gyrator structure of the lumped-winding component

of the core material be considered, the relative permeance $\mu_{r}$ will be a function of the magnetomotive force $M M F$, which is the magnetic "voltage" measured on the individual permeance. In linear case, $\mu_{r}$ can be simply configured as a constant.

After the core permeances get determined, only the value of the leakage permeances still remain undefined. Recalling that the nine type I divisions are identical in geometry and the transformer is symmetrical about the middle limb, yields the fact that only three parameters $P_{s}, P_{\text {long }}$ and $P_{\text {shunt }}$ are needed to characterise the whole leakage flux path, which are marked in Fig. 10. As has been discussed in the previous section, it is difficult to accurately define the geometry of the leakage flux path through the air, therefore a few short circuit tests are carried out to identify the three unknown parameters, in which one single winding is supplied by voltage source and selected others windings are short-circuited. Instead of enumerating all the supply-short combinations, only five shortcircuit test schemes are needed for the parameter identification which carry sufficient information about coupling between the windings. The equivalent inductance looking into the supplied winding is calculated as imaginary part of the measured complex impedance, as listed in TABLE II:

TABLE II: Test schemes for leakage permeance parameter fitting

\begin{tabular}{c|c|c|c}
\hline & Supply & Short & Fit to \\
\hline 1 & $W_{p}$ & $W_{s 1}$ & $L_{k 1, \text { Test }}=\operatorname{imag}\left(\vec{U}_{p} / \vec{I}_{p}\right) /(2 \pi f)$ \\
\hline 2 & $W_{p}$ & $W_{s 5}$ & $L_{k 2, \text { Test }}=\operatorname{imag}\left(\vec{U}_{p} / \vec{I}_{p}\right) /(2 \pi f)$ \\
\hline 3 & $W_{s 1}$ & $W_{s 2}$ & $L_{k 3, \text { Test }}=\operatorname{imag}\left(\vec{U}_{s 1} / \vec{I}_{11}\right) /(2 \pi f)$ \\
\hline 4 & $W_{s 1}$ & $W_{s 9}$ & $L_{k 4, \text { Test }}=\operatorname{imag}\left(\vec{U}_{s 1} / \vec{I}_{s 1}\right) /(2 \pi f)$ \\
\hline 5 & $W_{p}$ & All $W_{s} \mathrm{~s}$ & $L_{k 5, \text { Test }}=\operatorname{imag}\left(\vec{U}_{p} / \vec{I}_{p}\right) /(2 \pi f)$ \\
\hline
\end{tabular}

After that, the leakage permeance values of the circuit model in Fig. 10 are fitted to minimise a quadratic objective function, which is the square-summation of the error between the shortcircuit inductances from measurement and the one obtained from magnetic circuit simulation of the aforementioned 5 short-circuit schemes, given by the equation in below:

$$
f_{o b j}=\sum_{i=1}^{4}\left(\frac{L_{k i, T e s t}-L_{k i, S i m}}{L_{k i, T e s t}}\right)^{2}
$$

One should also note that the resistances of the windings need to be measured (e.g. using LRC-meter) and connected in series with the winding components in electrical circuit part of the model, if resistive part of the windings constitutes relatively large percentage of the short-circuit impedance, which is the case in the low voltage prototype. In case of high power medium voltage transformers however, inductive part will be much more dominated due to the large spacing between windings, so that accurate parametrising of the 


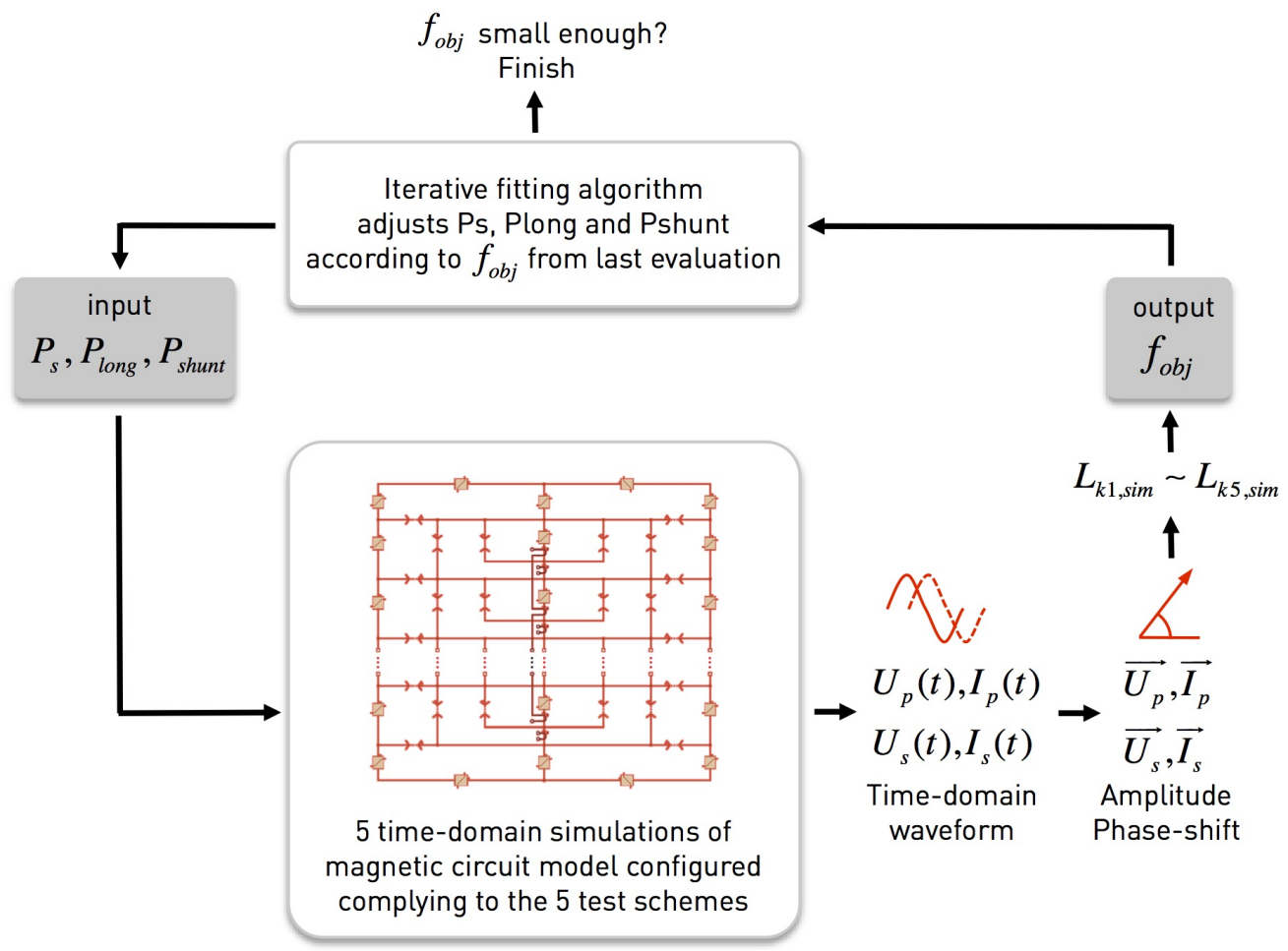

Fig. 12: Generalised fitting process of the leakage permeance values, where the magnetic circuit model is simulated to for calculating the output of the objective function for the fitting algorithm

winding resistance is not critical. Commonly used gradient based- or evolutionary algorithms can be applied to find out the leakage permeance values, iteratively. Generally the fitting process can be carried out in a way demonstrated in Fig. 12. On one side, after receiving the leakage permeance values $P_{s}, P_{\text {long }}, P_{\text {shunt }}$ provided by the fitting algorithm before each objective function evaluation, five magnetic circuit models of the transformer which are configured complying to each of the five test schemes specified by TABLE II are simulated in time-domain, one after each other. The simulated time-domain waveforms of winding voltage and current are transformed to amplitude and phase-angle, so as to calculate the equivalent inductance values and yields the output of the objective function following equation (2). On the other side, the fitting algorithm retrieves the output of objective function from the last evaluation, adjusts the $P_{s}, P_{\text {long }}, P_{\text {shunt }}$ values and brings them into the next objective function evaluation.

Initial values need to be assigned in order to make the algorithm better converge, which for the prototype transformer can be calculated in below. In circumstances the fitting algorithm may have difficulty in convergence if $P_{l o n g 0}$ is exactly equal to 0 , instead a small value of e.g. $10^{-9}$ can be chosen.

$$
\begin{gathered}
P_{s 0}=\frac{\mu_{r} \mu_{0} D_{c} d_{P S}}{h_{S}+h_{S S}} \\
P_{\text {long } 0}=0 \\
P_{\text {shunt } 0}=\frac{\mu_{r} \mu_{0} D_{c}\left(h_{S}+h_{S S}\right)}{d_{S C}}
\end{gathered}
$$

Despite of the repetitive structure, thanks to which the whole leakage flux path can be characterised by only three permeance values, strong unbalance of short-circuit impedances will still take place, which will be discussed in the following sections.

\section{VALIDATION WITH SHORT CIRCUIT TEST}

For verification of the proposed modelling's approach, the aforementioned prototype transformer has been realised in hardware. In order to carry out the five short-circuit schemes for model parameter identification and further schemes for validation, a test setup is established as shown in Fig. 13, which is composed of the following functional units:

- Power amplifier of type LM3866 to generate sinusoidal excitation supply voltage

- Shunt-type current sensor in combination with differential operational amplifier to accurately measure the current in both amplitude and phase-angle, referred to the supply voltage

- DC power supply $( \pm 16 \mathrm{~V})$ for the power amplifier and sensors

- Control unit (PLECS RT-Box) for reference signal generation for the power-amp and data acquisition

After the five inductance values listed in TABLE II have been obtained, the magnetic circuit models with the structure depicted in Fig. 10 is built up in the system-level simulation software PLECS (Blockset version) in combination with MATLAB/Simulink. Sinusoidal voltage sources are connected to the winding terminals to emulate the power amplifier output in the test setup. Prior to leakage parameter identification, the 


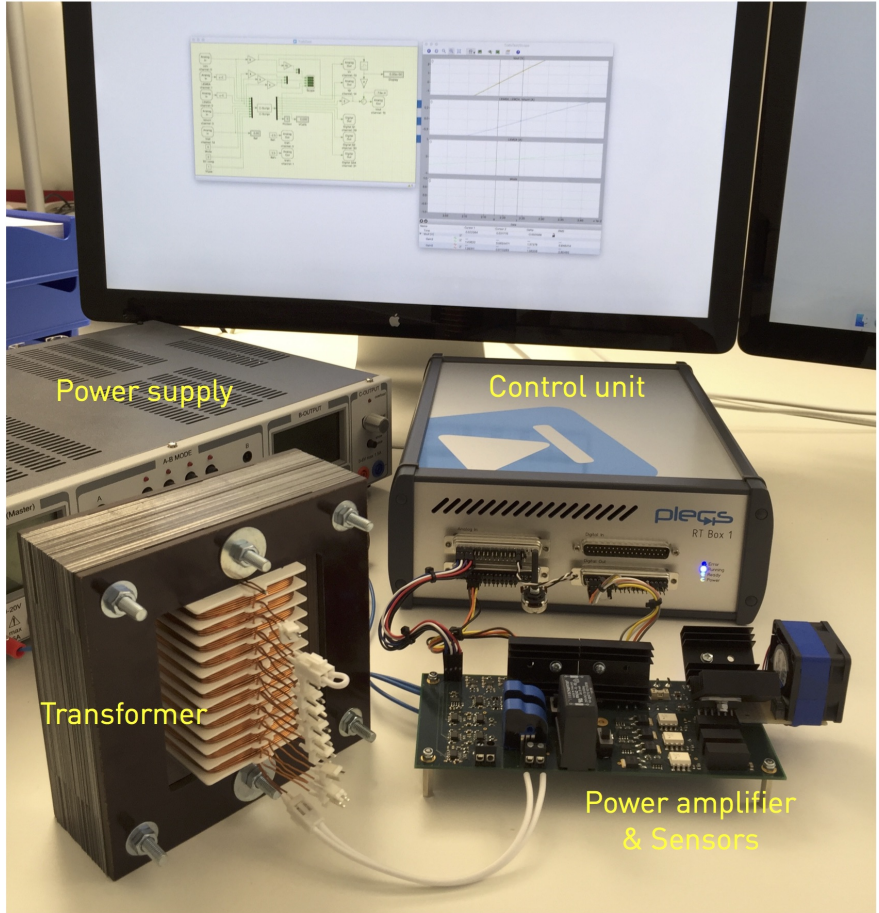

Fig. 13: Short-circuit test setup for the prototype transformer, with voltage and current on the supplied winding measured

permeances representing the iron core have been parametrised directly from geometry and material characteristic. Since the transformer is designed to operate far away from saturation, so that the nonlinearity of the core material has little affect on the measured short-circuit inductances, constant permeability from the data sheet has been assigned to the core permeances. The circuit model which is configured complying to test scheme 1 with primary winding $W_{p}$ supplied and secondary winding $W_{s 1}$ shorted is demonstrated in Fig. 14, while the ones for the other four test schemes are established in similar way. Also please note that the winding resistances can not be neglected

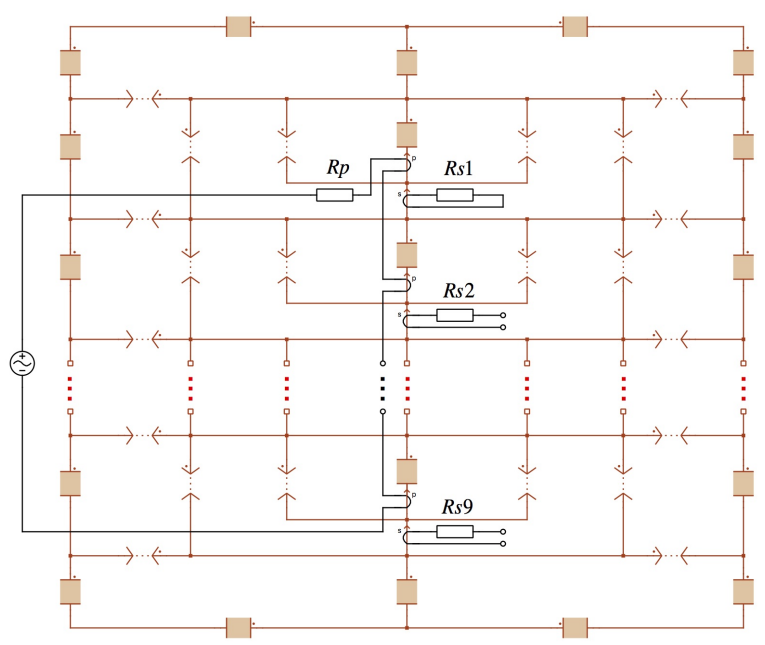

Fig. 14: Magnetic circuit model in PLECS for parameter fitting and verification and have been connected to the windings with $R_{p}=0.9 \Omega$ and $R_{s 1} \ldots R_{s 9}=0.15 \Omega$, considering the fact that the winding resistance is comparable to the measured short-circuit reactance, for example $X_{k 4, \text { Test }}=L_{k 4, \text { Test }} \cdot 2 \pi 400=0.10 \Omega$ is measured from test scheme 4 in TABLE II, which is $66 \%$ of the secondary winding resistance.

For parameter identification, the "fminsearch" fitting algorithm provided by MATLAB (referred as unconstrained nonlinear optimisation) is adopted. As required by "fminsearch", a customised objective function has been written using Matlab script which realises the functionalities listed in below:

- Accept the permeance values $\left(P_{s}, P_{\text {long }}, P_{\text {shunt }}\right)$ as input arguments and assign them to the five magnetic circuit models in PLECS/Simulink via mask variables.

- Simulate the five magnetic circuit models configured complying to each of the five test schemes specified in TABLE II, one by one.

- Calculate the equivalent inductance values $\left(L_{k 1, \text { Sim }} \cdots\right.$ $L_{k 5 \text {,Sim }}$ ) using the simulated voltage and current from the five models.

- Return the output of objective function calculated following equation (2).

The initial permeance values calculated using equation (3) till (5) as well as the ones obtained after parameter fitting using "fminsearch" algorithm are compared in TABLE III.

TABLE III: Leakage permeance values fitted to the measurements $[H]$

\begin{tabular}{l|c|c|c}
\hline & $P_{s}$ & $P_{\text {long }}$ & $P_{\text {shunt }}$ \\
\hline Initial value & $39 \cdot 10^{-9}$ & $1 \cdot 10^{-9}$ & $25 \cdot 10^{-9}$ \\
\hline After fitting & $81 \cdot 10^{-9}$ & $33 \cdot 10^{-9}$ & $32 \cdot 10^{-9}$ \\
\hline
\end{tabular}

In the first group of verification schemes, the primary winding is supplied, meanwhile one single secondary winding is shorted. The short-circuit inductances looking into the primary winding with different secondary windings shorted are compared between measurement and magnetic circuit model in Fig. 15. Although all the secondary windings have the same geometrical dimension and parameterised by repetitive permeance network, the resulted short-circuit impedances however, are unbalanced, due to difference in vertical position with respect to the primary winding. The U-shape curve reveals the fact that the more centralised the secondary winding is located with respect to the primary winding, the stronger is the coupling and thus the lower the short-circuit inductance will be.

In the second group of verification schemes, the secondary winding $W_{s 1}$ on the very top is supplied, meanwhile another secondary windings is shorted. The resulted inductances looking into $W_{s 1}$ are shown in Fig. 16 with different other secondary windings shorted. From the result comparison one can see that not only the points used for parameter identification (short $W_{s 1}, W_{s 5}$ in Fig. 15 and short $W_{s 2}, W_{s 9}$ in Fig. 16), but also the inductance values of the other verification points match quite well between the proposed magnetic circuit model (built in PLECS/Simulink) and test result, with maximum error of $8.3 \%$. 


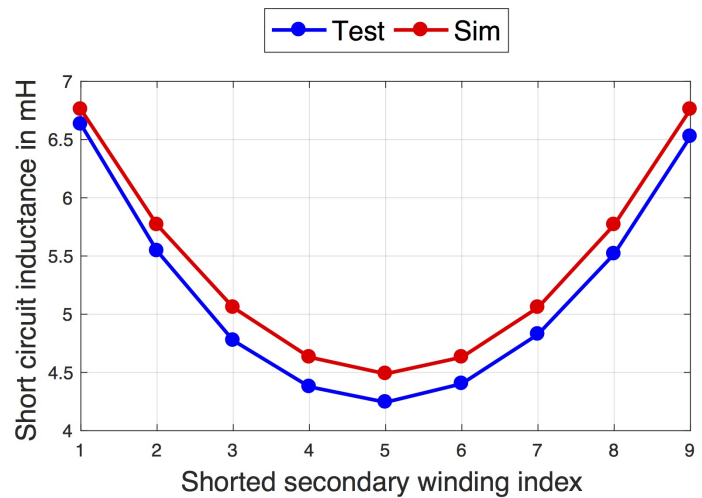

Fig. 15: Short circuit inductances with $W_{p}$ supplied and different single $W_{s}$ shorted

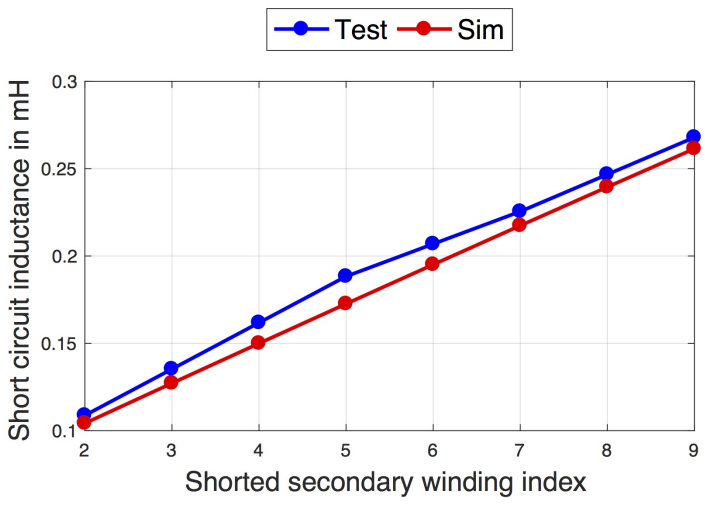

Fig. 16: Short circuit inductances with $W_{s 1}$ supplied and one of the other $W_{s}$ s shorted

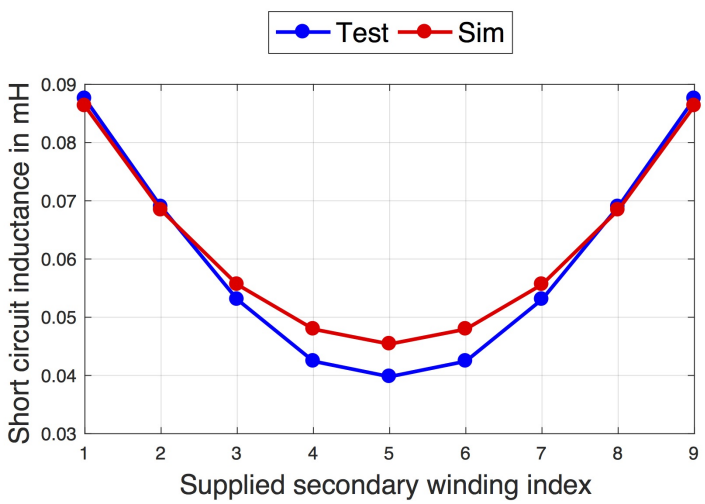

Fig. 17: Short circuit inductances with $W_{p}$ shorted and different single $W_{s}$ supplied

In the third group of verification schemes, the primary winding is shorted with single secondary winding supplied. The short-circuit inductances looking into the different secondary winding supplied are compared between simulation and test in Fig. 17. Maximum error of $12.0 \%$ occurs when $W_{s 5}$ is supplied.

In the forth group of verification schemes, one winding (primary or secondary) is supplied with other two shorted at the same time. The short-circuit combination together with the leakage inductance values looking into the supplied windings are listed in TABLE IV and TABLE V. Very good match between magnetic circuit model and the measurement is present here as well with maximum error of $5.0 \%$.

TABLE IV: Leakage inductances $[m H]$ comparison with one winding supplied and other two shorted: I

\begin{tabular}{c|c|c|c|c}
\hline Supplied & Shorted & $L_{k}$ Test & $L_{k}$ Sim & Error \\
\hline$W_{p}$ & $W_{s 1}, W_{s 2}$ & 4.03 & 3.85 & $4.5 \%$ \\
\hline$W_{s 1}$ & $W_{p}, W_{s 2}$ & 0.080 & 0.076 & $5.0 \%$ \\
\hline$W_{s 2}$ & $W_{p}, W_{s 1}$ & 0.058 & 0.059 & $1.7 \%$ \\
\hline
\end{tabular}

TABLE V: Leakage inductances $[m H]$ comparison with one winding supplied and other two shorted: II

\begin{tabular}{c|c|c|c|c}
\hline Supplied & Shorted & $L_{k}$ Test & $L_{k}$ Sim & Error \\
\hline$W_{p}$ & $W_{s 1}, W_{s 9}$ & 2.15 & 2.09 & $2.8 \%$ \\
\hline$W_{s 1}$ & $W_{p}, W_{s 9}$ & 0.093 & 0.095 & $2.2 \%$ \\
\hline$W_{s 9}$ & $W_{p}, W_{s 1}$ & 0.093 & 0.095 & $2.2 \%$ \\
\hline
\end{tabular}

\section{VALIDATION WITH POWER ELECTRONIC SYSTEM}

In this section, the fidelity of the proposed model in systemlevel simulation combined with power electronic converters is verified via experimental test. In verification scheme A the primary winding is excited by $115 \mathrm{~V}$ (rms), $400 \mathrm{~Hz}$ sinusoidal voltage generated from programmable AC source 61700 of Chroma, while five secondary windings $W_{s 1}, W_{s 6}, W_{s 7}, W_{s 8}$ and $W_{s 9}$ are connected to five totally identical full bridge rectifiers supplying constant resistive loads of $R_{L}=15 \Omega$, filtered by DC capacitors of $C_{d c}=3 m F$, as depicted in Fig. 18.

The system level simulation model has been established in PLECS/Simulink complying to the experimental setup, using the same transformer model discussed in section III, as Fig. 20 demonstrates. For the sake of fast system-level simulation,

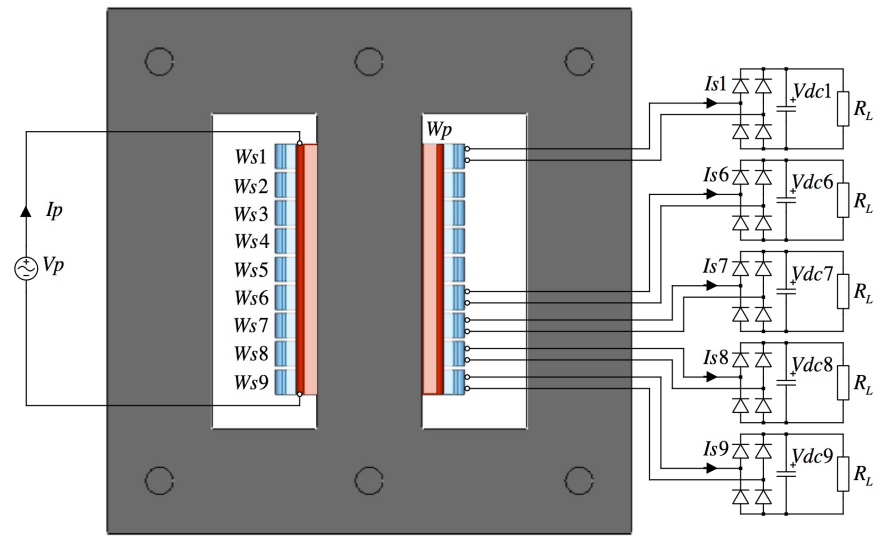

Fig. 18: Schematic of the system-level verification setup with five diode rectifiers connected (configured for verification scheme A with secondary windings $W_{s 1}, W_{s 6}$ till $W_{s 9}$ connected to rectifiers) 


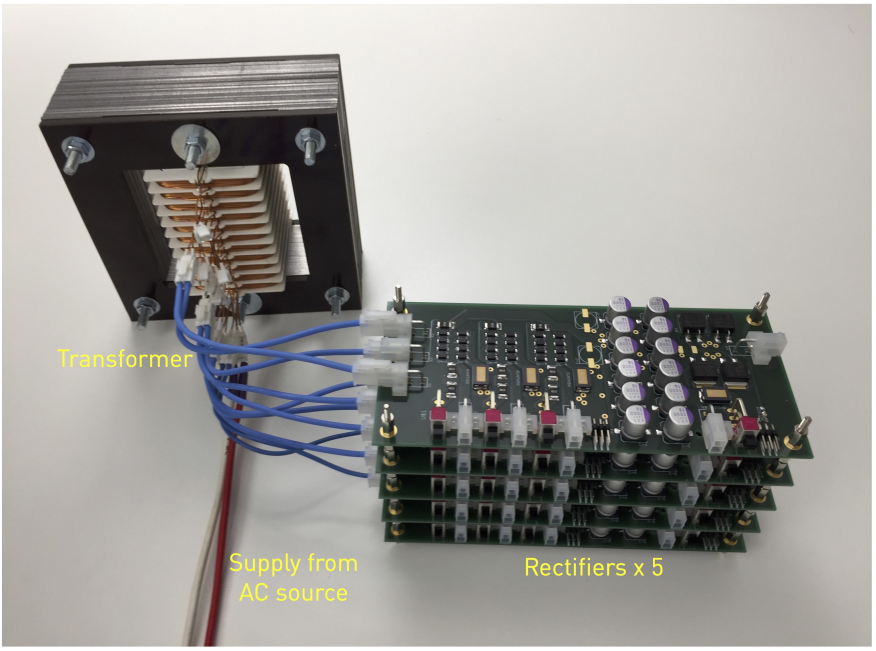

Fig. 19: Photo of the system-level verification setup

all the rectifier diodes are modelled as ideal switch in series connection with constant resistor, which represents the linearised forward characteristic obtained from manufacturer's data sheet. The secondary windings which are unconnected in the test setup are left as open circuit in the simulation model. The grid side voltage is modelled simply as an ideal sinusoidal voltage source without internal impedance, which corresponds to the terminal behaviour of the programmable AC source.

In Fig. 21 the simulated current on secondary windings $W_{s 1}, W_{s 6}$ and $W_{s 9}$ after parameter fitting as well as the ones directly using initial values obtained from equation (3) till (5) are compared to measurements from the experimental setup. The simulation result after parameter fitting is significantly closer to measurement than that with initial values. In TABLE VI quantitive comparison of the current RMS values is made, where the simulated result after parameter fitting presents a maximum error of only $1.3 \%$, while the ones using initial values has $8.0 \%$. This indicates that the fitting process which takes real measurement as reference is able to significantly

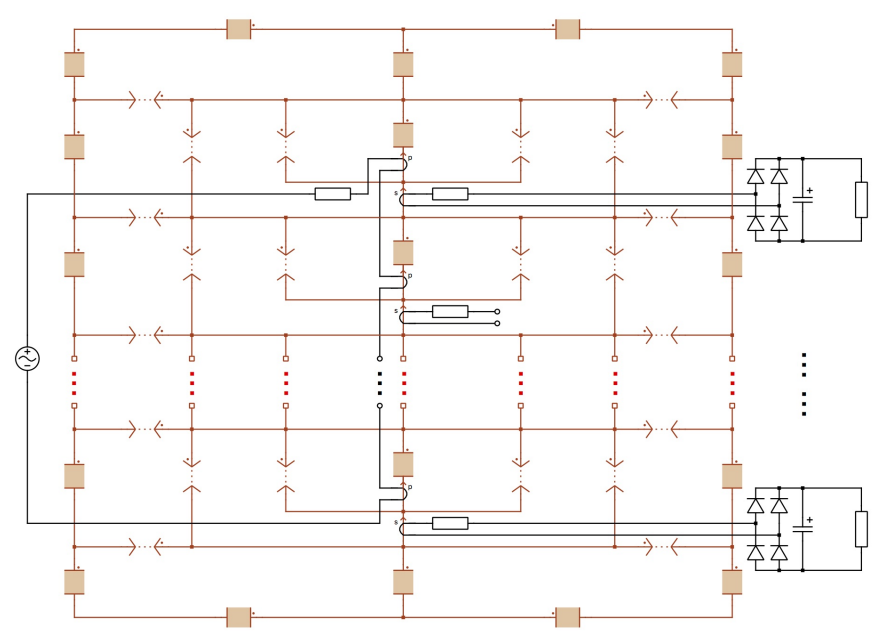

Fig. 20: System-level simulation model with diode rectifiers connected reduce the error of the model.

TABLE VI: RMS value of the secondary winding currents from measurement and simulation in verification scheme $\mathrm{A}$ [A]

\begin{tabular}{c|c|c|c}
\hline & $I_{s 1}$ & $I_{s 6}$ & $I_{s 9}$ \\
\hline Measured from experimental setup & 2.40 & 2.24 & 2.00 \\
\hline Simulated with fitted $P_{s}, P_{\text {long }}, P_{\text {shunt }}$ & 2.43 & 2.26 & 2.01 \\
\hline Simulated with initial $P_{s}, P_{\text {long }}, P_{\text {shunt }}$ & 2.59 & 2.37 & 2.10 \\
\hline
\end{tabular}

Further in Fig. 22 the current of the three secondary windings are displayed in the same plot and compared between measurement and simulation. Please note that although all the rectifiers are identically constructed and loaded, the current flowing on secondary winding $W_{s 9}$ however, is significantly lower in amplitude than that on $W_{s 1}$, also with obvious phase shift. In comparison, the current on $W_{s 5}$ closely resembles to that on $W_{s 1}$. The measured current on the other two secondary windings $W_{s 7}$ and $W_{s 8}$ are located just between $I_{s 5}$ and $I_{s 9}$ (not included into Fig. 22). This unbalance can be ascribed to the winding-position-related leakage flux coupling, which has been accurately captured by the proposed transformer model. Moreover, the magnetic circuit model allows to observe the flux distribution among the individual permeances, Fig. 23 demonstrates the simulated magnetic flux flowing through the leakage permeances of the type II magnetic circuit divisions belonging to $W_{s 1}, W_{s 6}$ and $W_{s 9}$, where strong unbalance is present.

TABLE VII: Verification schemes with different secondary windings connected to diode rectifiers

\begin{tabular}{c|c|c}
\hline Scheme & Supplied & Connected to diode rectifier \\
\hline $\mathrm{A}$ & $W_{p}$ & $W_{s 1}, W_{s 6}, W_{s 7}, W_{s 8}, W_{s 9}$ \\
\hline $\mathrm{B}$ & $W_{p}$ & $W_{s 1}, W_{s 5}, W_{s 6}, W_{s 7}, W_{s 8}$ \\
\hline $\mathrm{C}$ & $W_{p}$ & $W_{s 1}, W_{s 4}, W_{s 5}, W_{s 6}, W_{s 7}$ \\
\hline
\end{tabular}

The configuration of all verification schemes are listed in TABLE VII. In verification scheme $\mathrm{B}$, the first rectifier remains its connection to the secondary winding $W_{s 1}$, while the other four are shifted for one secondary winding upwards, that is, reconnected to the secondary windings $W_{s 5}$ till $W_{s 8}$. The current on the secondary windings $W_{s 1}, W_{s 5}$ and $W_{s 8}$ are compared again between measurement and simulation in Fig. 24 and Fig. 25 . Here the difference between secondary winding currents becomes smaller, in comparison to verification scheme A. Should the four rectifiers be shifted further upwards, the shape of secondary currents becomes nearly identical, (Fig. 26 and Fig. 27). Again the simulation model is able to capture the same behaviour of the experimental setup.

The results presented above demonstrate that the proposed model has the potential to reproduce the issues related to the unbalanced leakage flux distribution in system-level dynamic simulation. If customised to individual magnetic structures, this type of model may help engineers with improvement of control algorithm or proper selection of electrical components to compensate affects from the magnetic unbalance. Taking the verification case presented in this paper as example, since 

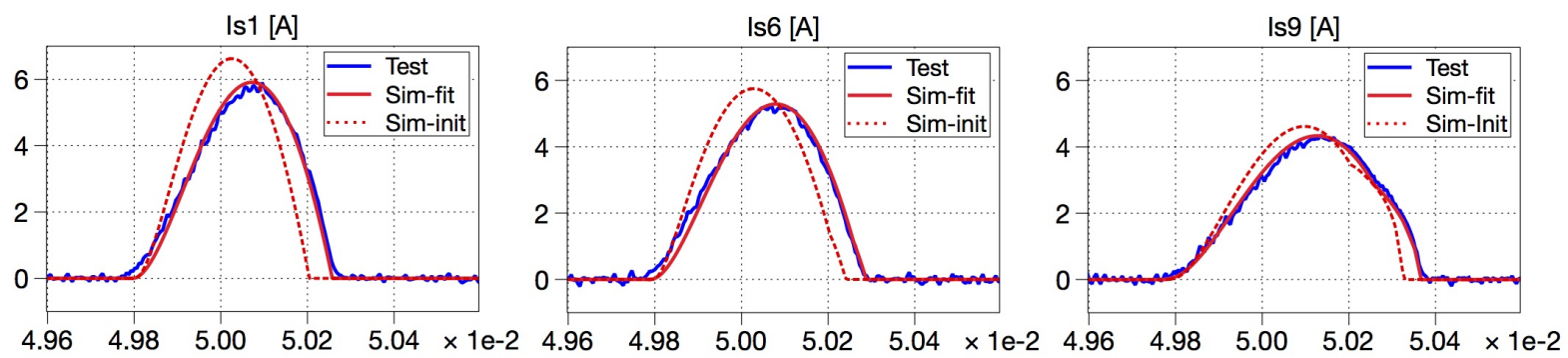

Fig. 21: Comparison of secondary currents in verification scheme A between measurement and simulation (solid curve), additionally to simulation result obtained with initial permeance values (dashed curve)

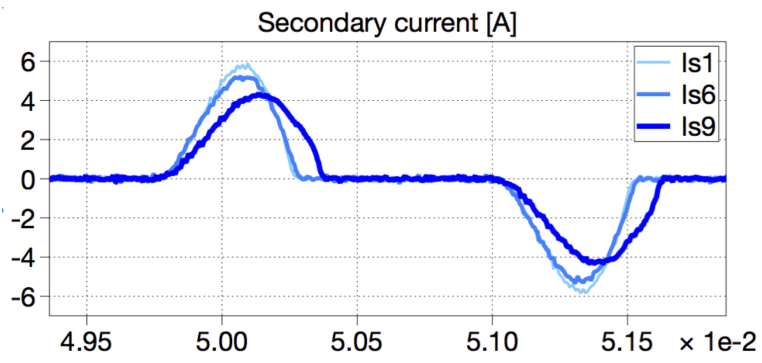

(a)

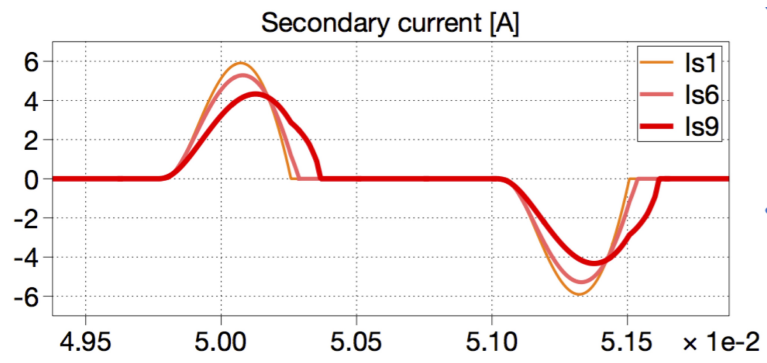

(b)

Fig. 22: Secondary winding currents in verification scheme A, with primary winding supplied, secondary windings $W_{s 1}, W_{s 6}$ till $W_{s 9}$ connected to rectifiers, (a) Test measurement (b) PLECS/Simulink simulation

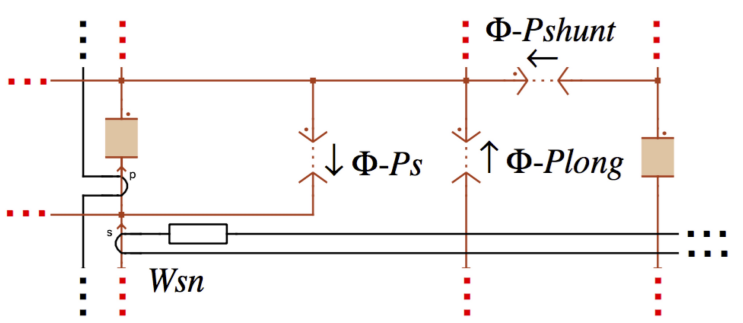

(a)

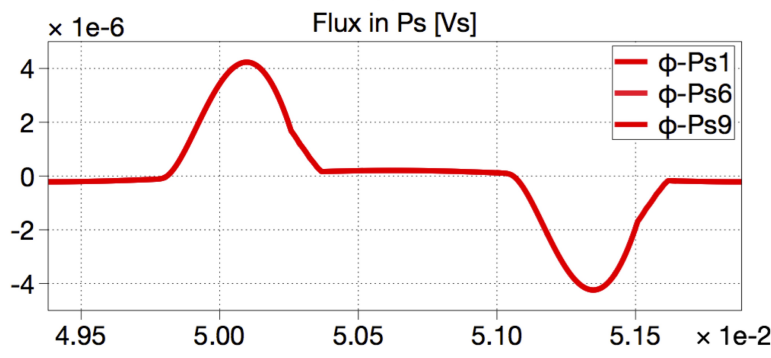

(c)

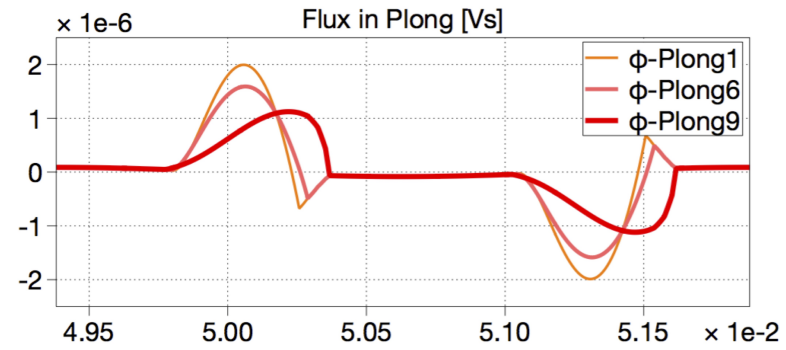

(b)

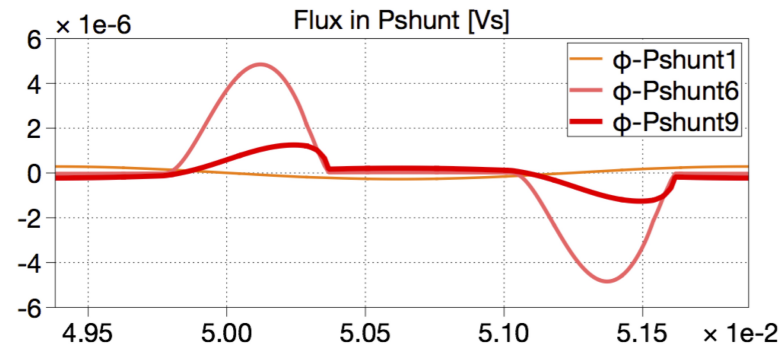

(d)

Fig. 23: Simulated flux in the magnetic circuit divisions including secondary windings $W_{s 1}, W_{s 6}$ and $W_{s 9}$ (a) Illustration of flux measurement positions in each magnetic circuit division (b) Simulated flux on $P_{l o n g}$ (c) Simulated flux on $P_{s}$ (d) Simulated flux on $P_{\text {shunt }}$

the rectifier input current is directly related to the ripple current of the DC-link capacitor, which strongly affect the capacitors' lifetime [22]. In practice it is desired to identify the ripple current in converter design-phase to select proper type of capacitors [23], for which the system-level simulation of the whole converter with the proposed transformer model integrated would provide a good reference.

\section{CONCLUSION}

This paper has demonstrated the modelling of multi-winding transformer using permeance-capacitance based magnetic circuit. The proposed model has the potential to reproduce circuit issues related to the unbalance in leakage flux coupling when integrated into system-level simulation combined with power converters. Via making use of information about the repetitive 

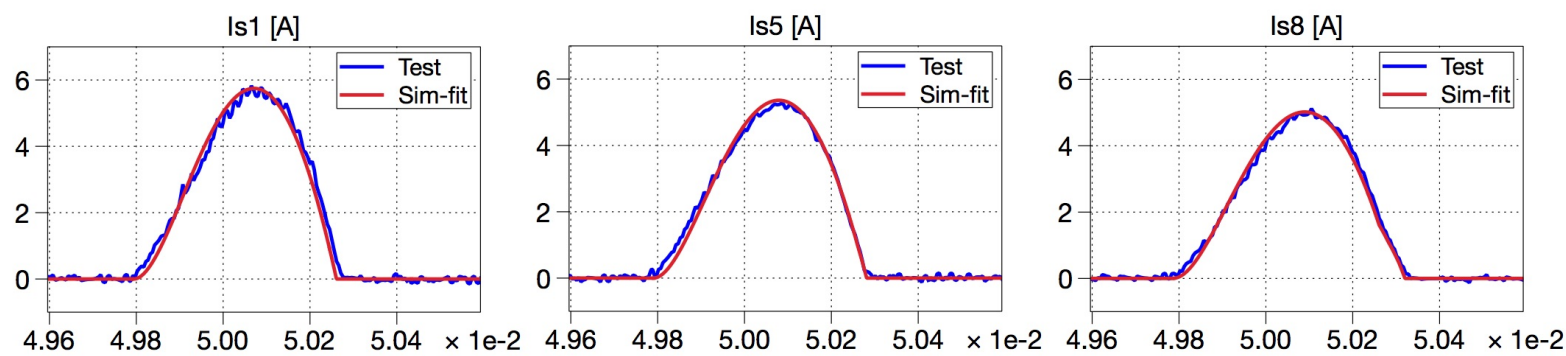

Fig. 24: Comparison of secondary currents in verification scheme B between measurement and simulation

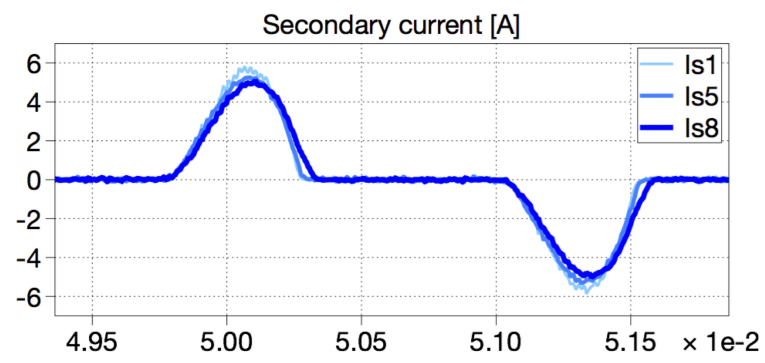

(a)

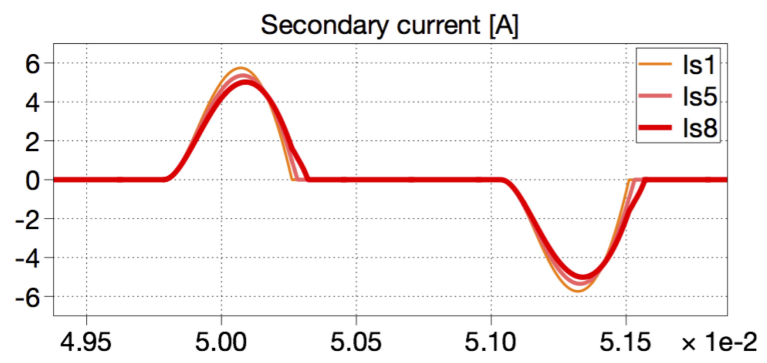

(b)

Fig. 25: Secondary winding currents in verification scheme B, with primary winding supplied, secondary windings $W_{s 1}, W_{s 5}$ till $W_{s 8}$ connected to rectifiers, (a) Test measurement (b) PLECS/Simulink simulation
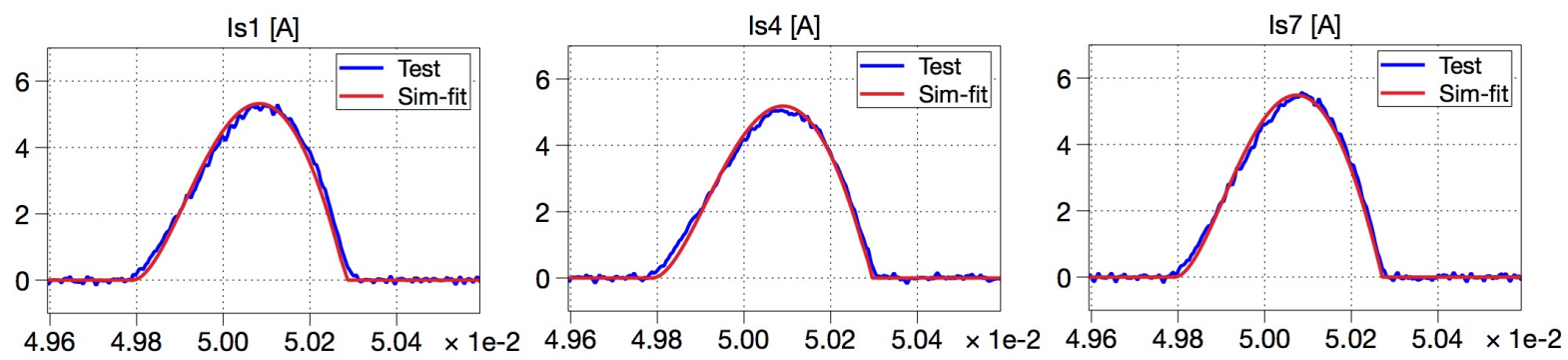

Fig. 26: Comparison of secondary currents in verification scheme $\mathrm{C}$ between measurement and simulation

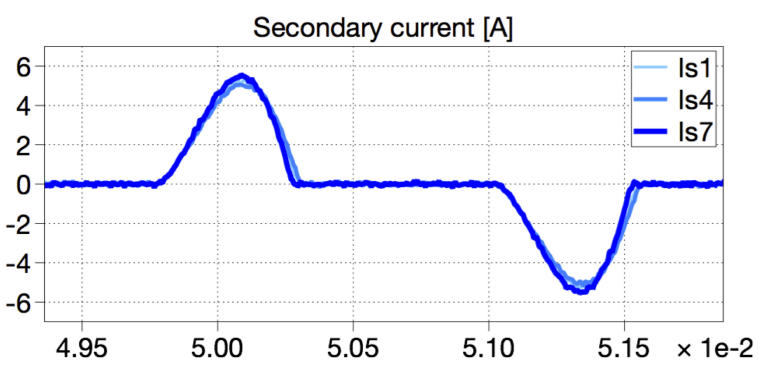

(a)

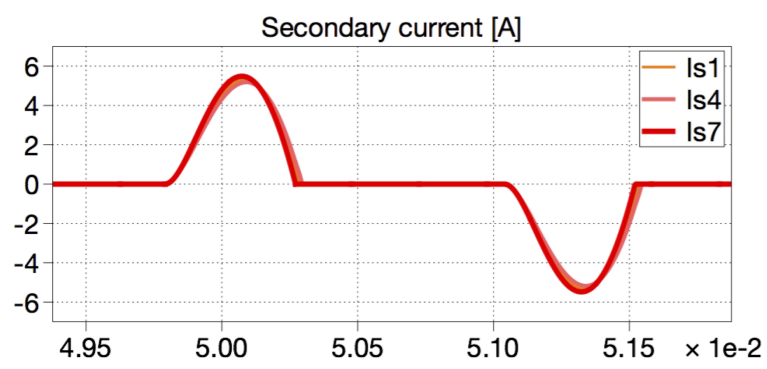

(b)

Fig. 27: Secondary winding currents in verification scheme C, with primary winding supplied, secondary windings $W_{s 1}, W_{s 4}$ till $W_{s 7}$ connected to rectifiers, (a) Test measurement (b) PLECS/Simulink simulation

and symmetrical geometry, the parameter identification process of the leakage flux path can be done from only a few experimental tests. The result from the proposed model shows good match to the hardware test, under a plenty of short-circuit schemes and in operation combined with power electronic converters. With the proposed model, effects that arise from the unbalanced leakage flux path could potentially identified during the design phase of the power converters. Future work will be invested in extending this approach to 3-phase phaseshifted transformers with different winding configurations, also fidelity of the model will be further verified via more transient cases e.g. during start-up of the power converter systems. 


\section{REFERENCES}

[1] Z. Zheng, Z. Gao, C. Gu, L. Xu, K. Wang, and Y. Li, "Stability and voltage balance control of a modular converter with multiwinding highfrequency transformer," in IEEE Transactions on Power Electronics, vol. 29 , no. 8, 2014, pp. 4183-4194.

[2] J. van Vlerken and P. G. Blanken, "Lumped modelling of rotary transformers, heads and electronics for helical-scan recording," in IEEE Transactions on Magnetics, vol. 31, no. 2, 1995, pp. 1050-1055.

[3] L. Guasch, F. Corcoles, and J. P. L. Sainz, "Effects of symmetrical voltage sags on three-phase three-legged transformers," in IEEE Transactions on Power Delivery, vol. 19, no. 2, 2004, pp. 875-883.

[4] A. Davoudi, P. L. Chapman, J. Jatskevich, and A. Khaligh, "Reducedorder modeling of high-fidelity mangetic equivalent circuits," in IEEE Transactions on Power Electronics, vol. 24, no. 12, 2009, pp. $2847-$ 2855.

[5] A. D. Theocharis, J. Milias-Argitis, and T. Zacharias, "Three-phase transformer model including magnetic hysteresis and eddy currents effect," in IEEE Transactions on Power Delivery, vol. 24, no. 3, 2009, pp. 1284-1294.

[6] P. S. Moses, M. A. S. Masoum, and H. A. Toliyat, "Dynamic modeling of three-phase asymmetric power transformers with magnetic hysteresis: no-load and inrush conditions," in IEEE Transactions on Energy Conversion, vol. 25, no. 4, 2010, pp. 1040-1047.

[7] P. G. Blanken, "A lumped winding model for use in transformer models for circuit simulation," in IEEE Transactions on Power Electronics, vol. 16, no. 3, 2001, pp. 445-460.

[8] J. Cale, S. D. Sudhoff, and L.-Q. Tan, "Accurately modeling ei core inductor using a high-fidelity magnetic equivalent circuit approach," in IEEE Transactions on Magnetics, vol. 42, no. 1, 2006, pp. 40-46.

[9] C. Alverez-Marino, F. de Leon, and X. M. Lopez-Fernandez, "Equivalent circuit for the leakage inductance of multiwinding transformers: unification of terminal and duality models," in IEEE Transactions on Power Delivery, vol. 27, no. 1, 2012, pp. 353-361.

[10] S. Jazebi and F. de Leon, "Experimentally validated reversible singlephase multiwinding transformer model for the accurate calculation of low-frequency transients," in IEEE Transactions on Power Delivery, vol. 30, no. 1, 2015, pp. 193-201.

[11] L. Cao and J. Yang, "Linear circuit model of the trhee-phase insulated core transformer power supply," in IEEE Transactions on Nuclear Science, vol. 63, no. 1, 2016, pp. 288-296.

[12] F. de Leon and J. A. Martinez, "Dual three-winding transformer equivalent circuit matching leakage measurements," in IEEE Transactions on Power Delivery, vol. 24, no. 1, 2009, pp. 160-168.

[13] M. Lambert, M. Matinez-Duro, J. Mahseredjian, F. de Leon, and F. Sirois, "Transformer leakage flux models for electromagnetic transients: Critical review and validation of a new model," in IEEE Transactions on Power Delivery, vol. 29, no. 5, 2014, pp. 2180-2188.

[14] M. Lambert, F. Sirois, M. Matinez-Duro, and J. Mahseredjian, "Analytical calculation of leakage inductance for low-frequency transformer modelling," in IEEE Transactions on Power Delivery, vol. 28, no. 1, 2013, pp. 507-515.

[15] M. Luo, D. Dujic, and J. Allmeling, "Leakage flux modelling of multiwinding transformer using permeance magnetic circuit," in 2016 IEEE Applied Power Electronics Conference and Exposition (APEC), 2016, pp. $1108-1114$

[16] B. Azzerboni, E. Cardelli, G. Finocchio, and F. L. Foresta, "Remarks about preisach function approximation using lorentzian function and its identification for nonoriented steels," in IEEE Transactions on Magnetics, vol. 39, no. 5, 2003, pp. 3028-3030.

[17] M. Luo and D. Dujic, "Permeance based modelling of the core corners considering magnetic material nonlinearity," in Annual Conference of the IEEE Industrial Electronics Society (IECON), 2015, pp. 950-955.

[18] P. W. Hammond, "A new approach to enhance power quality for medium voltage ac drives," in IEEE Transactions on Industry Applications, vol. 33, no. 1, 1997, pp. 202-208.

[19] D. Hamill, "Lumped equivalent circuits of magnetic components: the gyrator-capacitor approach," in IEEE Transactions on Power Electronics, vol. 8, 1994, pp. 97-103.

[20] J. Allmeling, W. Hammer, and J. Schönberger, "Transient simulation of magnetic circuits using the permeance-capacitance analogy," in Control and Modeling for Power Electronics (COMPEL), IEEE 13th Workshop on, 2012.

[21] E. C. Snelling, Soft Ferrite: Properties and Applications. London Iliffe Books Ltd., 1969 .
[22] M. A. Vogelsberger, T. Wiesinger, and H. Ertl, "Life-cycle monitoring and voltage-managing unit for dc-link electrolytic capacitors in pwm converters," in IEEE Transactions on Power Electronics, vol. 26, no. 2, 2011, pp. 493-503.

[23] L. Sun, Z. Wu, W. Ma, X. Fei, X. Cai, and L. Zhou, "Analysis of the dc-link capacitor current of power cells in cascaded h-bridge inverters for high-voltage drives," in IEEE Transactions on Power Electronics, vol. 29 , no. 12 , 2014, pp. 6281-6292.

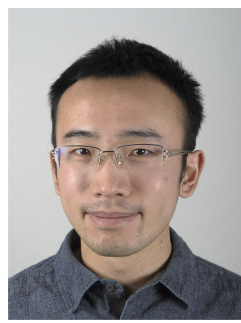

Min Luo Min Luo (M'13) was born in Beijing, China, in 1986. He received the B.S. degree in electrical engineering from Tsinghua University, Beijing, China, in 2009 and M.S. degree in electrical power engineering from RWTH Aachen University, Aachen, Germany, in 2012. Since 2012 he has been with Plexim as application engineer, working on the software PLECS for fast simulation of power electronic systems. In 2014 he joined power electronics laboratory of École Polytechnique Fédérale de Lausanne (EPFL) in Lausanne, Switzerland as external doctoral research assistant, pursuing his $\mathrm{PhD}$ degree. His current research interest include dynamic modelling of magnetic components and real-time HIL simulation of power converters.

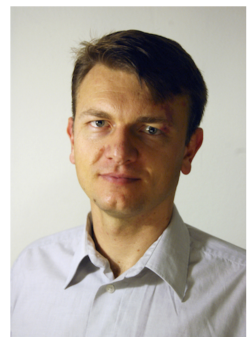

Drazen Dujic Drazen Dujic (S'03-M'09-SM'12) received the Dipl.-Ing. and M.Sc. degrees from the University of Novi Sad, Novi Sad, Serbia, in 2002 and 2005, respectively, and the Ph.D. degree from the Liverpool John Moores University, Liverpool, U.K., in 2008, all in electrical engineering. From 2002 to 2006, he was with the Department of Electrical Engineering, University of Novi Sad as a Research Assistant, and from 2006 to 2009 with Liverpool John Moores University as a Research Associate. From 2009 till 2013, he was with ABB Corporate Research Centre, Switzerland, as a Principal Scientist working on the power electronics projects spanning the range from low-voltage/power SMPS in below kilowatt range to medium voltage high-power converters in a megawatt range. During 2010-2011, he was a member of a project team responsible for the development of the worlds first power electronic traction transformer (PETT) successfully commissioned on the locomotive. From 2013 till 2014, he was with ABB Medium Voltage Drives, Turgi, Switzerland, as R\&D Platform Manager, responsible for ABB's largest IGCT based medium voltage drive - ACS6000. He is currently with Ecole Polytechnique Federale de Lausanne EPFL, Lausanne, Switzerland, as an Assistant Professor and the Director of the Power Electronics Laboratory. His current research interests include the areas of design and control of advanced high-power electronics systems and high performance drives. He has authored or coauthored more than 80 scientific publications and has filed eleven patents. He is an Associate Editor for IEEE Transactions on Industrial Electronics, IEEE Transaction on Power Electronics and IET Electric Power Applications. He has received the First Prize Paper Award by the Electric Machines Committee of the IEEE Industrial Electronics Society at IECON-2007. In 2014 he has received the Isao Takahashi Power Electronics Award for outstanding achievement in power electronics.

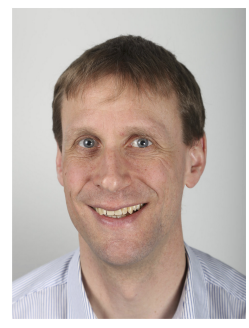

Jost Allmeling Jost Allmeling (S'98-M'03) was born in Hamburg, Germany, in 1972. He received the M.S. degree in electrical engineering from Aachen University, Aachen, Germany, in 1996 and the Ph.D. degree from the Swiss Federal Institute of Technology (ETH), Zurich, Switzerland, in 2001. In 1996, he became a Research Associate at the Power Electronics Laboratory at ETH. From 2001 until 2003, he was with the Power Systems Laboratory at ETH, as a Post-Doctoral Researcher. In 2002, Jost co-founded Plexim, a spin-off company from ETH Zurich that develops the software PLECS for fast simulation of power electronic systems. Currently, he is the Managing Director of Plexim. His research interests include simulation of power electronics, modeling electrical and mechanical components, real-time HIL simulation and inverter control. 